\title{
Thinking in Parallel: foreword
}

\section{Vicente Matellán Olivera ${ }^{1}$. José Luis González-Sánchez ${ }^{2}$}

Published online: 6 May 2021

(c) The Author(s), under exclusive licence to Springer Science+Business Media, LLC, part of Springer Nature 2021

Under the title of Supercomputing Education: Thinking in Parallel, this special issue of the Journal of Supercomputing (SUPE) was promoted from the TEEM'19 International Conference ${ }^{1}$ held in León (Spain) in October 2019. A special session under the same title was organized at the Conference, focused on the factors that have to be taken into account for improving training in Supercomputing, on identifying the limitations of Supercomputing training, and on how to provide solutions for these limitations.

The use of Supercomputers is wide spreading, constituting an essential component in many fields of science. The interest in the use of high-performance computing (HPC) facilities is also increasing in a growing percentage of undergraduates because the use of these infrastructures allows them to improve their skills and the results of their training. For this reason, the demand of training on supercomputing increases continuously. "Thinking in Parallel" intended to focus on the factors that have to be taking into account for improving training in Supercomputing for improving the performance of researchers and also to try to identify the limitations of current supercomputing training and to provide solutions for these limitations.

In particular, this special issue is focused on experiences and tools to facilitate the parallelization of existing code, training of researchers and professionals with nonIT backgrounds, recycling of IT professional for the HPC ecosystem, and learning methodologies, tools, and experiences for training of computer science students in parallel programming.

The call for papers ended in January 2020 and the topics of interest were experiences and tools to facilitate the parallelization of existing code, tools for training of

\footnotetext{
${ }^{1}$ https://2019.teemconference.eu.
}

Vicente Matellán Olivera

vicente.matellan@scayle.es

José Luis González-Sánchez

joseluis.gonzalez@cenits.es

1 Fundación Centro de Supercomputación de Castilla y León (SCAYLE), León, Spain

2 Centro Extremeño de iNvestigaciónInnovación Tecnológica y Supercomputación (CénitS), Cáceres, Spain 
researchers and professionals with non-IT backgrounds on the use of supercomputing, recycling of IT professional for the HPC ecosystem, learning methodologies, tools, and experiences for training of computer science students in parallel programming, and evaluation of the learning process.

Seventeen proposals were received, two of them were refused by guest editors, and the rest of them went through a rigorous review process in the middle of the pandemic caused by COVID-19. Six papers have finally been accepted from the remaining 15 reviewed. Of the six accepted papers, four of them focus their research on training in supercomputing environments and two are more specifically focused on helping researchers using supercomputing to think and work in parallel.

The work of Corral-García et al. presents a transcompiler for helping researchers and inexperienced users, who do not have the necessary skills in the use of parallel programming. Its effectiveness is analyzed and discussed in work through a complete set of tests to measure and evaluate benefits achieved when applying these techniques.

A different approach has been taken in Guerrero-Higueras et al.'s approach for teaching students parallel programming using a different application program interface: OpenMP, MPI, CUDA, or OpenCL. Authors demonstrated that the direct interaction of students through the Gitter tool has a positive impact on the learning process.

The rest of the paper has shown a different approach. The most relevant research in many fields is currently based on the use of HPC facilities, so, very different domains have been presented to the special issue, from Quantum to Ethological studies. Experiences, tool, and methodologies have proposed.

In the case of quantum computing teaching presented by Carrascal et al., comparison and tests of different quantum programming environments have been faced. Qualitative and quantitative analyses have been performed, focusing on cross-platform parameters such as qubits, circuit depth, number of gates, and code design effort. By taking these analyses into account, a methodology for teaching quantum computing is proposed which includes the use of OOP to program a basic quantum simulator, a quantum circuit test with a graphical interface, the programming of real quantum computers with a programming language, and a deep exploration of known quantum algorithms.

The need of skilled professionals in the efficient use of these infrastructures means that some reforms in education need to be considered with the aim of providing a good training previously to the end of the high education stage. Early acquisition of these skills is the foundation for developing higher and more complex skills in a future, which are needed to drive creativity and innovation.

In this way, Fernández et al.'s paper studies the interaction between STEM (Science, Technology, Engineering, and Mathematics) and non-STEM subjects for developing Supercomputing training activities, in order to improve the performance of non-STEM students attending courses related to Supercomputing.

A more specific approach of this problem has been analyzed in Bence et al.'s paper, where ethologist first approach to HPC is analyzed taken into account the cultural differences between two different European countries. 
The design of a learning and teaching framework committed to train masters' students in Big Data capable of automatically tracking students' progress, while effectively exploiting the diversity of their backgrounds, and assisting the teaching staff is proposed in Solé-Beteta et al.'s article.

Finally, as guest editors, we would like to specially thank the Journal of Supercomputing's Editor-in-Chief as well as all the external reviewers for their invaluable support and help in the preparation and publication of this special issue.

Publisher's Note Springer Nature remains neutral with regard to jurisdictional claims in published maps and institutional affiliations. 